Roy G. M. Kolkman • Wiendelt Steenbergen •

Ton G. van Leeuwen

\title{
In vivo photoacoustic imaging of blood vessels with a pulsed laser diode
}

Received: 7 March 2006 / Accepted: 30 March 2006 / Published online: 24 May 2006

(C) Springer-Verlag London Limited 2006

\begin{abstract}
Photoacoustic imaging is a hybrid imaging modality that is based on the detection of acoustic waves generated by absorption of pulsed light by tissue chromophores such as hemoglobin in blood. For this technique, usually large and costly Q-switched Nd:YAG lasers are used. These lasers provide a pulse energy of at least several milliJoules. In search of alternative light sources, we investigated the use of a small and compact pulsed laser diode to image blood vessels. We proved that a pulsed laser diode can be applied for imaging blood vessels in vivo.
\end{abstract}

Keywords Imaging techniques · Ultrasound ·

Blood vessels

\section{Introduction}

In recent years, a broad interest is present in developing new techniques for noninvasive imaging of blood vessels and blood containing structures in tissue, such as tumors. Photoacoustic imaging (PAI), also called optoacoustic imaging, is an ideal candidate because this hybrid technique is based on the detection of acoustic waves generated by the absorption of pulsed light by tissue chromophores such as hemoglobin in blood. PAI exploits the high intrinsic contrast of optical imaging and the spatial resolution of pure ultrasound imaging.

In PAI, the tissue is illuminated by a short light pulse (typical pulse length of approximately $10 \mathrm{~ns}$ ), which is partly absorbed at positions where hemoglobin (blood), is

R. G. M. Kolkman $(\bowtie) \cdot$ W. Steenbergen · T. G. van Leeuwen Biophysical Engineering, Institute for BioMedical Technology, Faculty of Science and Technology, University of Twente, P.O. Box 217, Enschede 7500 AE, The Netherlands e-mail: r.g.m.kolkman@utwente.nl

Tel.: +31-53-4893164

Fax: $+31-53-4891105$

T. G. van Leeuwen

Laser Center, Academic Medical Center,

University of Amsterdam,

P.O. Box 22700, Amsterdam 1100 DE, The Netherlands present. A local adiabatic temperature rise will occur, followed by a pressure rise due to restricted dilatation. As a result of the initial pressure rise, a pressure transient will propagate through the tissue, which subsequently can be detected at the surface. From the time of flight of the pressure transient, i.e., the time between firing the laser and the detection of the pressure transient, the distance of the photoacoustic source to the detector can be calculated using knowledge of the speed of sound in tissue.

Next to this qualitative approach, the amplitude and the shape of the photoacoustic signal can also be analyzed quantitatively. First, in the case that the condition of stress confinement is fulfilled, the initial pressure rise will be proportional to the absorbed energy. This condition requires that there is insignificant relaxation of pressure in the region of light absorption during the laser pulse, which means that the pulse duration $t_{\mathrm{p}}$ has to be smaller than the propagation time of the pressure transient through the region (size $d$ ) with speed of sound $c: t_{\mathrm{p}}<d / c$. The size of region $d$ is determined by its smallest dimension or by the optical penetration depth that is equal to $1 / \mu_{\mathrm{eff}}$, with $\mu_{\mathrm{eff}}$ being the effective optical attenuation coefficient. Secondly, the temporal profile of the pressure transient will be dependent on the source geometry [1]. For spherical and cylindrical sources, this temporal profile will have a typical bipolar shape, in which the distance between the two peaks is dependent on the size of the source [2].

Due to its intrinsic optical absorption contrast and its quantitative nature, PAI has successfully been applied to in vivo imaging of blood vessel-structures in small animals [3-8] and humans [9-12]. Recently, we have shown that the diameter of blood vessels in vitro as well as in vivo can be estimated from the peak-to-peak time (time between the occurrence of the positive and negative peaks) of the bipolar pressure transient [2]. In addition, we showed that venous valves can be identified with photoacoustic imaging [13]. Furthermore, the application of PAI in mammography is being investigated [14-16].

Until now, large pulsed laser systems, such as Qswitched Nd:YAG lasers have been used for PAI. These lasers produce pulses with a typical duration of $10 \mathrm{~ns}$ and 
several milliJoules per pulse at a wavelength of $1,064 \mathrm{~nm}$ (fundamental) or $532 \mathrm{~nm}$ (frequency doubled). In addition, combinations of Nd:YAG lasers with an optical parametric oscillator (OPO) were used to obtain wavelengths in the near-infrared with similar pulse energies and pulse durations. A step towards miniaturizing the PAI system will be to use small and relatively cheap pulsed laser diodes. Recently, the feasibility of the use of pulsed laser diodes in PAI has been explored in phantom studies [17]. In this paper, the feasibility of the use of a pulsed laser diode for in vivo imaging is investigated. Results are presented from experiments on phantoms as well as human superficial blood vessels.

\section{Materials and methods}

\section{Pulsed lasers}

In this research, the use of a pulsed laser diode module (iRLS, Laser Components GmbH, Germany) was investigated and results were compared with the PA signals induced by a Nd:YAG laser + OPO (Opolette, Opotek, USA). The pulsed laser module generated pulses with a duration of $112 \mathrm{~ns}$ and a peak power of $210 \mathrm{~W}$ at a wavelength of $905 \mathrm{~nm}$ with a pulse repetition rate of $5 \mathrm{kHz}$ and a maximum pulse energy of $23 \mu \mathrm{J}$. The size of this module was $46.5 \times 33.5 \times 12 \mathrm{~mm}$. The $\mathrm{Nd}: \mathrm{YAG}+\mathrm{OPO}$ laser, tunable from 690 to $950 \mathrm{~nm}$, was operated at a wavelength of $905 \mathrm{~nm}$ with a pulse duration of $7 \mathrm{~ns}$, a repetition rate of $20 \mathrm{~Hz}$ and an energy of about $3-5 \mathrm{~mJ} /$ pulse.

When the condition of stress confinement is fulfilled, the amplitude of the photoacoustic signal is proportional to the amount of energy that is absorbed by the blood and, thus, proportional to the laser pulse energy. As the pulse energy of the laser diode is significantly lower than the energy per pulse of the conventional Nd:YAG laser, more averages are required to obtain the same signal-to-noise ratio (SNR). In our experiments, the energy per pulse of the pulsed-laser diode is approximately a factor of 100 lower than that of the Nd:YAG laser. Because the SNR scales with the square root of the number of averages, the number of pulses used for averaging in the experiments with the pulsed laser diode module has to be $100^{2}=10,000$ times larger than the number of pulses when using the Nd:YAG laser to obtain an equivalent SNR.

\section{Photoacoustic imaging}

The light of the pulsed lasers was coupled into a glass fiber (ø $600 \mu \mathrm{m}, \mathrm{NA}=0.22$ ) that was integrated in a home-built double ring photoacoustic sensor. This double-ring sensor consisted of two concentric ring-shaped electrodes with equal areas $[9,10]$. This sensor had an angular aperture of $1.5-10^{\circ}(-6 \mathrm{~dB}$ of directivity pattern) for acoustic signals with a peak-to-peak time of 67-350 ns, respectively. Due to this narrow angular aperture, the measured time traces could be regarded as one-dimensional depth images [amplitude (A)-scans] of photoacoustic sources inside the measurement volume. The sensor was mounted on a translation stage to enable scanning, which allowed us to obtain a 2D dataset (scan direction vs depth) of PA signals. To visualize the contours of the vessels, the absolute values of the A-scans were plotted in a 2D-image plane, using a logarithmic gray scale. This procedure is shown schematically in Fig. 1. To reduce the contribution of noise, a threshold at two times the rms value of the noise was applied, making all signals below this threshold appearing as black in the images.

The cross-section of the vessels can be reconstructed by fitting a bipolar signal (derivative of a Gaussian) to the measured photoacoustic pressure transients. Next, the resulting fit was integrated over depth (time) to obtain the absorption distribution. This absorption distribution corresponds with the cross-section of the blood vessel [10].

\section{Experiments}

\section{Phantom measurements}

The in vitro experiments were performed on an artificial blood vessel made of a nylon tube (inner diameter $1 \mathrm{~mm}$ ), filled with flowing dye. A dilution of Ecoline Black (Royal Talens, the Netherlands) was used as dye that had an absorption coefficient of $0.6 \mathrm{~mm}^{-1}$ at $905 \mathrm{~nm}$, which is comparable to the absorption of blood at this wavelength. For this absorption coefficient and diameter of the artificial vessel, the condition of stress confinement is fulfilled for both lasers and, thus, the amplitude of the pressure transient is proportional to the absorbed energy. The expected peakto-peak time of the pressure transient for this artificial vessel can be calculated [2] from the diameter and yields 338 ns.

The artificial vessel was placed in a container filled with water. Photoacoustic imaging was carried out with the pulsed laser diode module with an energy out of the fiber of $4.4 \mu \mathrm{J} / \mathrm{pulse}$ at a pulse repetition rate of $5 \mathrm{kHz}$. The photoacoustic signals were digitized by a dual channel oscilloscope-card (NI PCI-5122, 100 Msample/s, $100 \mathrm{MHz}$
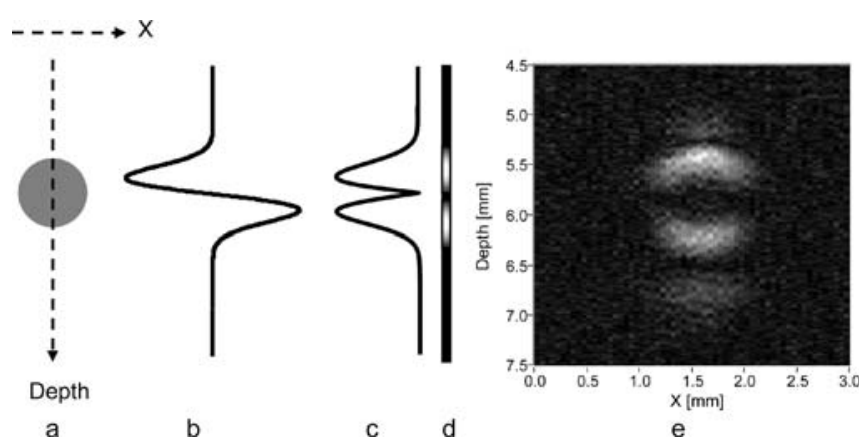

Fig. 1 Photoacoustic imaging a sensor is scanned over blood vessel; b time trace (A-scan) at position above vessel, time is converted to depth by multiplying time with speed of sound; $\mathbf{c}$ rectified time trace; $\mathbf{d}$ conversion to grayscale; $\mathbf{e}$ repeating $\mathbf{a}-\mathbf{d}$ as a function of $X$ position results in a photoacoustic image 
bandwidth, National Instruments). A 2D set of A-scans of the artificial vessel was obtained by 61 A-scans, equidistantly positioned with $0.05 \mathrm{~mm}$ spacing, along a line, resulting in a total B-scan time of 3 min. Each A-scan consisted of 10,000 averages.

The results of the experiment with the pulsed laser diode were compared with the results obtained with the Nd:YAG laser + OPO with a pulse energy of $0.45 \mathrm{~mJ} /$ pulse. In this case, no averaging of the individual A-scans was applied. In this way, it is expected that for both measurements a similar signal to noise ratio is obtained. The total B-scan time for the $61 \mathrm{~A}$-scans using the Nd:YAG laser + OPO was $32 \mathrm{~s}$.

The signal-to-noise ratio (SNR) was analyzed by measuring 50 times a time trace at a position above the center of the vessel and averaging the SNRs of all these time traces. The SNR was defined as the peak-to-peak voltage (voltage difference between maximum value of positive peak and minimum value of negative peak) of the photoacoustic pressure transient divided by the rms voltage of the noise.

\section{In vivo measurements}

Experiments were performed with the pulsed laser diode module on blood vessels at the posterior side of the hand of a volunteer. Acoustic coupling between the tissue surface and photoacoustic sensor was obtained by using an optically transparent ultrasound contact gel (Sonogel, Bad Camberg, Germany).

Acoustic signals were generated by illuminating the hand with $7.3 \mu \mathrm{J} /$ pulse at a pulse repetition rate of $5 \mathrm{kHz}$, with a wavelength of $905 \mathrm{~nm}$ and pulse duration of $112 \mathrm{~ns}$. The radiant exposure at the skin was about $0.2 \mathrm{~mJ} / \mathrm{cm}^{2}$ per pulse.

A 2D set of A-scans of superficial blood vessels at the posterior side of the hand was obtained by 51 A-scans, equidistantly positioned with $0.1 \mathrm{~mm}$ spacing, along a line, resulting in a total B-scan time of $3 \mathrm{~min}$. A second scan was made at another position, consisting of 51 subsequent Ascans with a spacing of $0.075 \mathrm{~mm}$. Each A-scan consisted of 10,000 averages.

\section{Results}

\section{Phantom measurements}

In Fig. 2, the time traces for both lasers are shown at a position above the center of the vessel $(X=1.5 \mathrm{~mm}$ in Fig. 3). The diameter of the vessel was estimated from the peak-to-peak time [2]. This resulted in a diameter of $0.95 \mathrm{~mm}$ for both measurements.

In Fig. 3, photoacoustic images of the cross section of the nylon tube filled with ecoline are displayed. In image A, the photoacoustic reconstruction is shown when a pulsed Nd:YAG laser is used to generate the photoacoustic

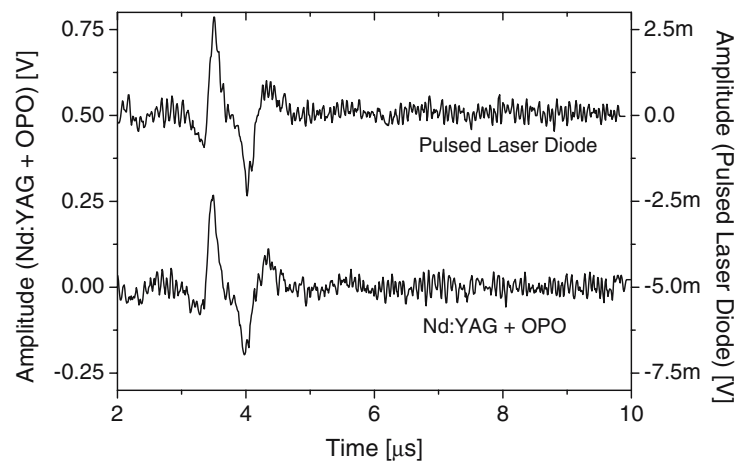

Fig. 2 Photoacoustic time trace of the images in Fig. 3 for both the pulsed laser diode (right vertical axis, average of 10,000 pulses of $4.4 \mu \mathrm{J}$ each) and the Nd:YAG laser $+\mathrm{OPO}$ (left vertical axis, single $0.45 \mathrm{~mJ}$ pulse) at position $X=1.5 \mathrm{~mm}$

signals; in image $\mathrm{B}$, the resulting reconstruction is shown for a pulsed laser diode.

Analysis of SNR ( \pm standard deviation) for the pulsed laser diode and the Nd-YAG laser OPO system at position $X=1.5 \mathrm{~mm}$ (Fig. 3) yielded values of $25.3 \pm 1.8$ and $26.2 \pm$ 2.5 , respectively. According to the Student's $t$ test with a $95 \%$ confidence interval these two values are significantly different $(p=0.038)$.
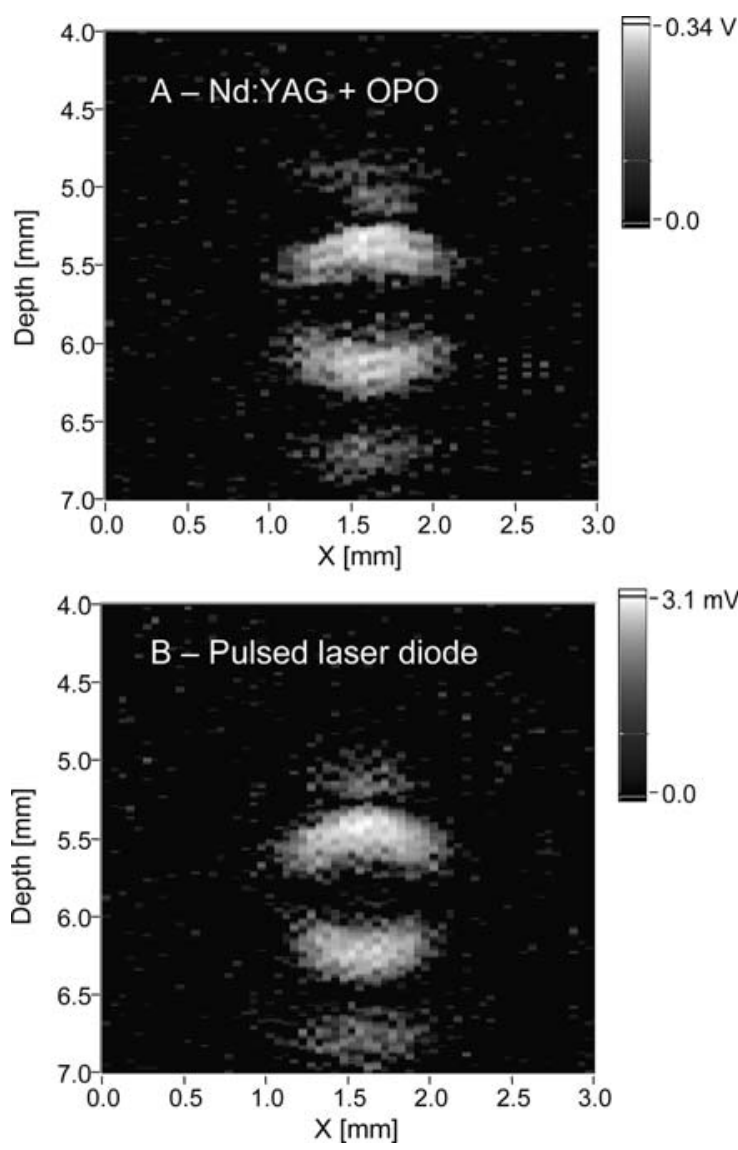

Fig. 3 Photoacoustic reconstruction of a nylon tube filled with Ecoline-Black surrounded by water: a pulsed Nd:YAG laser $+\mathrm{OPO}$, $0.45 \mathrm{~mJ} /$ pulse, no averaging; b pulsed laser diode $4.4 \mu \mathrm{J} /$ pulse, 10,000 averages per time trace (A-scan) 


\section{In vivo measurements}

In Fig. 4, photoacoustic images of blood vessels at the posterior side of the hand are shown. In image a, a vessel is visualized with a diameter of about $1 \mathrm{~mm}$, located $1 \mathrm{~mm}$ below the skin. In image $b$, two vessels, close to each other, are visualized, both with a diameter of about $0.6 \mathrm{~mm}$, located $1 \mathrm{~mm}$ below the skin. In images $\mathrm{c}$ and $\mathrm{d}$, the reconstructed absorption distributions are visualized, showing the cross section of the blood vessels and the contour of the skin.

\section{Discussion}

In this paper, the use of a pulsed laser diode in photoacoustic imaging of superficial human blood vessels was demonstrated. 2D images of the cross section of vessels located at $1 \mathrm{~mm}$ below the skin were obtained.

The time traces for both pulsed-laser diode and Nd:YAG laser appear very similar (Fig. 2). The diameter of the artificial vessel was estimated from the peak-to-peak time and was $0.95 \mathrm{~mm}$ which is close to the expected diameter of $1 \mathrm{~mm}$. Analysis of SNR for the pulsed laser diode and
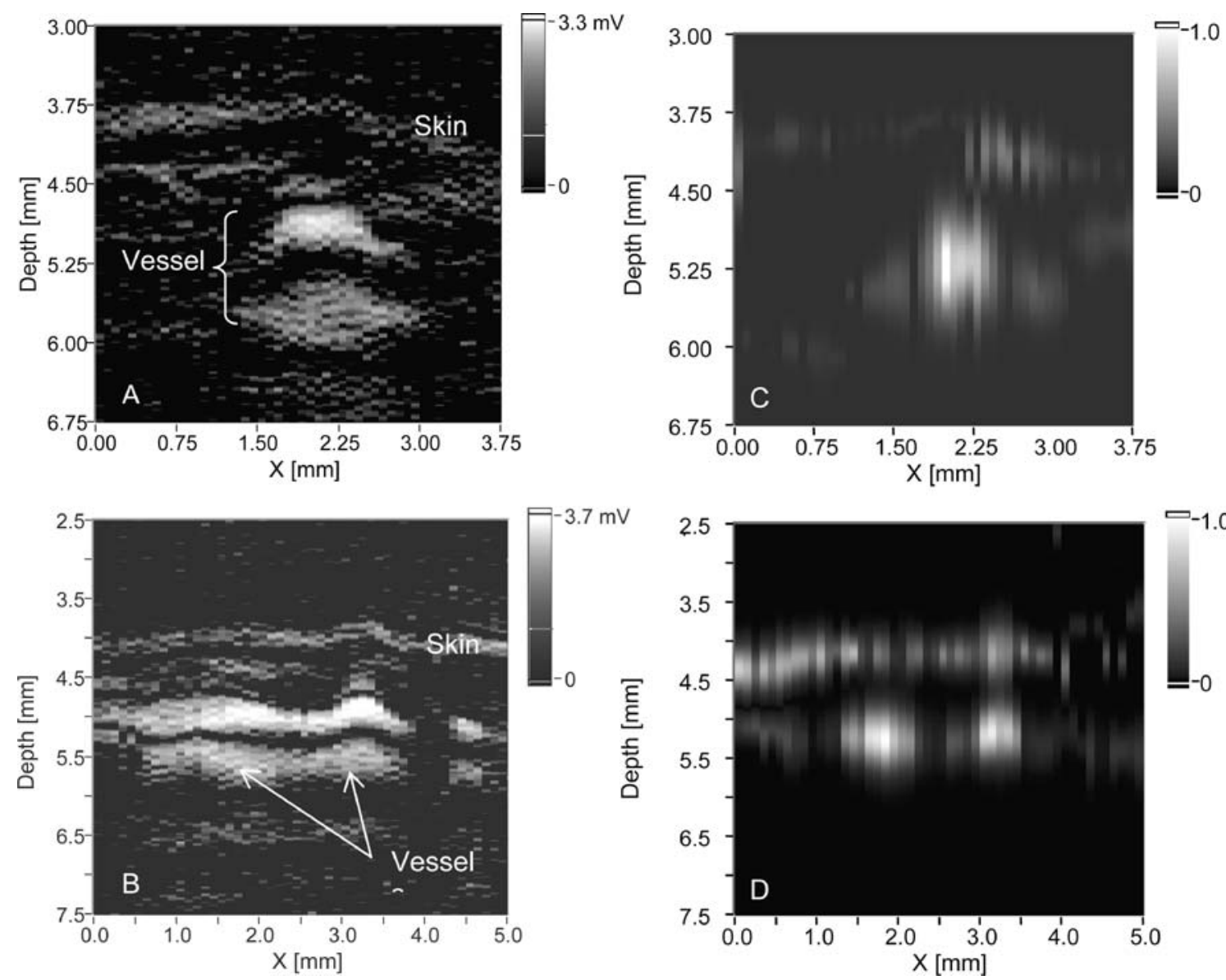

Fig. 4 a Photoacoustic reconstruction of a vessel at proximal side of the hand; b photoacoustic reconstruction of two vessels close to each other at proximal side of the hand. Photoacoustic signals were generated with a pulsed laser diode with a pulse energy of $7.3 \mu \mathrm{J} /$ the Nd-YAG laser OPO system yielded values of $25.3 \pm 1.8$ and $26.2 \pm 2.5$, respectively. According to the Student's $t$ test these two values were significantly different. However, the small difference in SNR between these two measurements can be explained by the ratio between the pulse energies not being exactly 100: correction for the exact ratio of pulse energies on the assumption of proportionality between signal amplitude and pulse energy, yields identical SNR values for both lasers.

The in vivo measurements of superficial blood vessels at the posterior side of the hand (Fig. 4) show that these vessels can be visualized with photoacoustic imaging using a pulsed laser diode.

The pulse duration of the pulsed laser diode (112 ns) is much longer than the pulse duration of the $\mathrm{Nd}$ :YAG laser (7 ns). In case of the pulsed laser diode, the condition of stress confinement will then no longer be fulfilled for small blood vessels (i.e., smaller than $173 \mu \mathrm{m}$, assuming a speed of sound in tissue of $1,540 \mathrm{~m} / \mathrm{s}$ ). As a consequence, the initial pressure rise for sources smaller than $173 \mu \mathrm{m}$ will be smaller compared to a 7-ns pulse and the resulting pressure transient will be broadened in time. This implies that also the resolution of the system will be limited by this pulse duration. However, the measurements shown in this paper

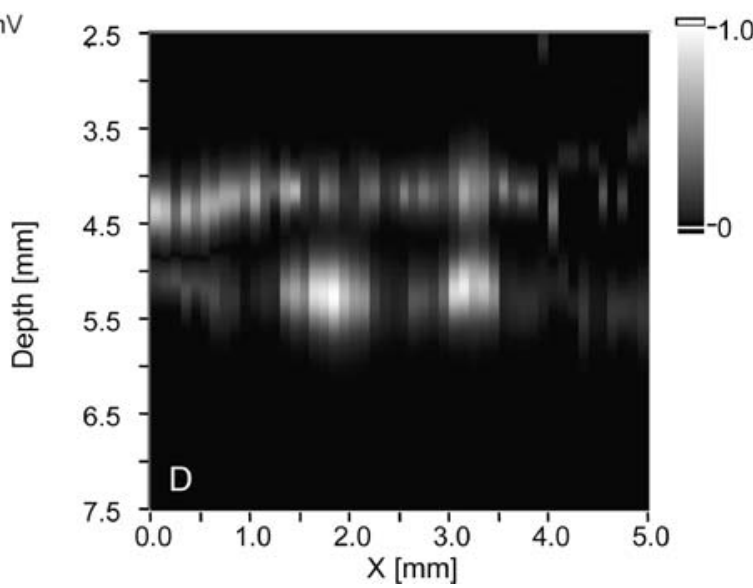

pulse. $\mathbf{c}$ and $\mathbf{d}$ Reconstruction of the absorption distribution by fitting a bipolar signal to the measured pressure transients and integrating this fitted signals over depth 
are not affected by this longer pulse duration as the artificial as well as in vivo vessels had a diameter much larger than $173 \mu \mathrm{m}$.

A feature of the pulsed laser diodes is the limited amount of energy per pulse compared to most Nd:YAG lasers. This leads to a large number of averages for a given desired SNR, implying an increase in measurement time. This can be overcome by further increasing the repetition rate or by using multiple laser diodes to increase the pulse energy.

In a comparison of the performances of the diode laser and the Nd:YAG laser for photoacoustic imaging, the combined effects of pulse repetition rate, pulse energy, and the exposure time on potential harm of the laser light to the skin have to be taken into account. Although laser safety regulations cannot directly be translated in maximum exposure limits for patients, these limitations can be used as a guide for determining the optimal laser parameters from the point of view of laser safety. Using the IEC laser safety standard [18] as a guideline, the maximum permitted exposure [MPE, $\left.\left(\mathrm{J} / \mathrm{m}^{2}\right)\right]$ for skin decreases for an increasing number of administered pulses as well as for an increasing repetition rate (paragraph 13.3 of [18]). This will affect the SNR that can be obtained in a certain measurement time. When applying the maximum pulse energy as permitted by the safety standard for a given repetition rate and number of pulses $N$, the amplitude of the photoacoustic pressure transient due to a single laser pulse will scale proportional to the pulse energy related to the MPE. In addition, the noise-amplitude when averaging over $N$ pulses will scale with $1 / \sqrt{ } N$. In Fig. 5, the improvement in SNR due to averaging is shown as a function of total time needed to measure $N$ pulses for a pulse repetition rate of 20 and $5 \mathrm{kHz}$ (pulse duration 10-100 ns, wavelength $905 \mathrm{~nm}$ ). This improvement in SNR is calculated with respect to the SNR of a single pulse. The SNR of a single pulse is equal for both repetition rates as the MPE for a single pulse is not dependent on the repetition rate. In case of a repetition rate

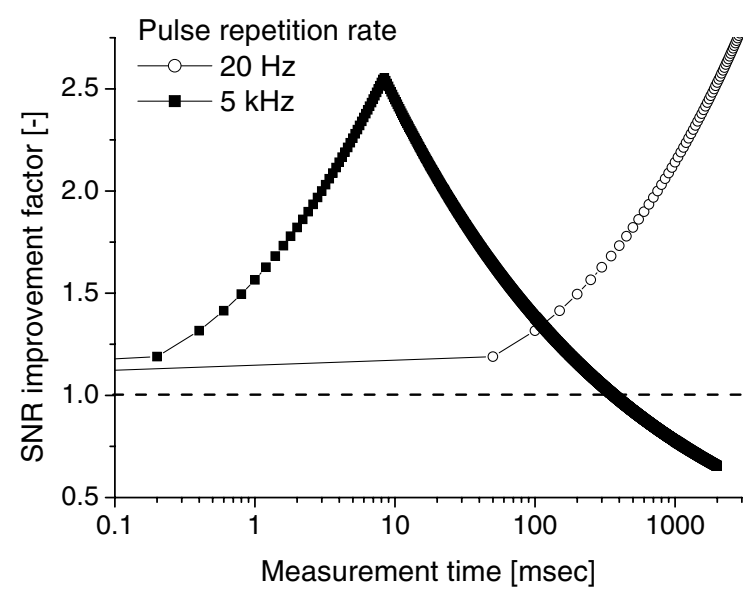

Fig. 5 Improvement in SNR with respect to the SNR of a single pulse, as a function of required measurement time (repetition rate $\times$ number of averages) for a pulse repetition rate of 20 and $5 \mathrm{kHz}$ (pulse duration 10-100 ns, $905 \mathrm{~nm}$ ). SNR is calculated by scaling the signal amplitude to the maximum permitted exposure (MPE) according to laser safety standard IEC 60825-1 of $20 \mathrm{~Hz}$, the SNR improves when averaging over an increasing number of pulses. In case of a repetition rate of $5 \mathrm{kHz}$, there is a maximum increase in SNR at 43 pulses. For a larger number of pulses, the improvement in SNR decreases due to limitation of the pulse energy by the laser safety standard because of the $5 \mathrm{kHz}$ repetition rate, resulting in a SNR less than the SNR obtained for a single pulse after about 1,800 pulses. This implies that increasing the number of averages while observing the laser safety regulations not always results in an improvement of SNR. When designing a system based on a pulsed laser diode or another high-repetition rate laser system, one has to optimize the system parameters such as repetition rate and number of averages by taking into account the MPE which affects the SNR that can be obtained.

As the pulsed laser diode is much more compact as well as one order of magnitude cheaper than a Nd:YAG laser, this allows for the development of compact photoacoustic scanners for imaging superficial vessels. Furthermore, these laser diodes are available at several different wavelengths. This allows for photoacoustic spectroscopy in which the amplitude of the pressure transient is measured as a function of wavelength to reveal information of the spectral dependence of the absorption coefficient. This can, for instance, be applied to determine the local oxygen saturation in the tissue.

\section{Conclusions}

The use of pulsed laser diodes in photoacoustic imaging of superficial human blood vessels has been demonstrated. It was proven that blood vessels at a depth of about $1 \mathrm{~mm}$ below the skin could be imaged using a pulsed laser diode module providing a pulse energy on the tissue of $7.3 \mu \mathrm{J} /$ pulse.

Acknowledgements This work was partly funded by the Netherlands Foundation of Fundamental Research on Matter FOM (grant 00PMT22) and the Institute for BioMedical Technology (BMTI) of the University of Twente.

\section{References}

1. Hoelen CGA, de Mul FFM (1999) A new theoretical approach to photoacoustic signal generation. J Acoust Soc Am 106:695706

2. Kolkman RGM, Klaessens JHGM, Hondebrink E, Hopman JCW, de Mul FFM, Steenbergen W, Thijssen JM, van Leeuwen TG (2004) Photoacoustic determination of blood vessel diameter. Phys Med Biol 49:4745-4756

3. Wang XD, Pang YJ, Ku G, Xie XY, Stoica G, Wang LV (2003) Noninvasive laser-induced photoacoustic tomography for structural and functional in vivo imaging of the brain. Nat Biotechnol 21:803-806

4. Wang XD, Pang YJ, Ku G, Stoica G, Wang LV (2003) Threedimensional laser-induced photoacoustic tomography of mouse brain with the skin and skull intact. Opt Lett 28:1739-1741 
5. Siphanto RI, Thumma KK, Kolkman RGM, van Leeuwen TG, de Mul FFM, van Neck JW, van Adrichem LNA, Steenbergen W (2005) Serial noninvasive photoacoustic imaging of neovascularization in tumor angiogenesis. Opt Express 13:89-95

6. Siphanto RI, Kolkman RGM, Huisjes A, Pilatou MC, de Mul FFM, Steenbergen W, van Adrichem LNA (2004) Imaging of small vessels using photoacoustics: an in vivo study. Lasers Surg Med 35:354-362

7. Kruger RA, Kiser WL, Reinecke DR, Kruger GA (2003) Thermoacoustic computed tomography using a conventional linear transducer array. Med Phys 30:856-860

8. Kruger RA, Kiser WL, Reinecke DR, Kruger GA and Miller KD (2003) Thermoacoustic optical molecular imaging of small animals. Mol Imaging 2:113-123

9. Kolkman RGM, Hondebrink E, Steenbergen W, de Mul FFM (2003) In vivo photoacoustic imaging of blood vessels using an extreme-narrow aperture sensor. IEEE J Sel Top Quantum Electron 9:343-346

10. Kolkman RGM, Hondebrink E, Steenbergen W, van Leeuwen TG, de Mul FFM (2004) Photoacoustic imaging with a doublering sensor featuring a narrow aperture. J Biomed Opt 9:13271335

11. Niederhauser JJ, Jaeger M, Lemor R, Weber P, Frenz M (2005) Combined ultrasound and optoacoustic system for real-time high-contrast vascular imaging in vivo. IEEE Trans Med Imaging 24:436-440

12. Viator JA, Choi B, Ambrose M, Spanier J, Nelson JS (2003) In vivo port-wine stain depth determination with a photoacoustic probe. Appl Opt 42:3215-3224
13. Bosschaart N, Kolkman RGM, van Leeuwen TG, Steenbergen W (2006) Imaging of venous valves with photoacoustics. In: Oraevsky AA, Wang LV (eds) Photons plus ultrasound: imaging and sensing 2006, Proceedings of SPIE vol 6086, SPIE Bellingham (in press)

14. Kruger RA, Kiser WL Jr, Romilly AP, Schmidt P (2001) Thermoacoustic CT of the breast: pilot study observations. In: Oraevsky AA (ed) Biomedical Optoacoustics II, Proceedings of SPIE vol 4256, SPIE Bellingham, pp 1-5

15. Manohar S, Kharine A, van Hespen JCG, Steenbergen W, van Leeuwen TG (2005) The Twente photoacoustic mammoscope: system overview and performance. Phys Med Biol 50:25432557

16. Oraevsky AA, Andreev VG, Karabutov AA, Solomatin SV, Savateeva EV, Fleming RD, Gatalica Z, Singh H (2001) Laser optoacoustic imaging of breast cancer in vivo. In: Oraevsky AA (ed) Biomedical Optoacoustics II, Proceedings of SPIE vol 4256, SPIE Bellingham, pp 81-94

17. Allen TJ, Cox BT, Beard PC (2005) Generating photoacoustic signals using high-peak power pulsed laser diodes. In: Oraevsky AA, Wang LV (eds) Photons plus ultrasound: Imaging and sensing 2005, Proceedings of SPIE vol 5697, SPIE Bellingham, pp 233-242

18. International standard IEC 60825-1:1993+A1:1997+A2 (2001) Safety of laser products-Part 1: Equipment classification, requirements and user's guide, IEC Geneva, Switzerland 